\title{
Degradación química de pet y su metabolismo por consorcios fúngicos
}

\section{Chemical degradation of pet and its metabolism by fungal consortia}

\author{
DOI: $10.46932 / \operatorname{sfjdv3n1-114}$
}

Received in: Jan 30st, 2021

Accepted in: Feb 1th, 2022

\section{Leticia Guadalupe Navarro Moreno}

Profesor Investigador

Institucion: Instituto de Química, Universidad del Papaloapan

Direccíon: Circuito Central 200, Parque Industrial, 68301 San Juan Bautista Tuxtepec, Oax. México

E-mail: navarroleticia483@gmail.com

\section{Andrea Rangel Cordero}

Analista

Departamento de Microbiología del Instituto Nacional de Ciencias Médicas y Nutrición Salvador

Zubirán. Ciudad de México

E-mail: ayleen.cardenaso@incmnsz.mx

\section{Cirilo Nolasco Hipólito}

Profesor Investigador

Institucion: Instituto de Biotecnología, Universidad del Papaloapan.

Direccíon: Circuito Central 200, Parque Industrial, 68301 San Juan Bautista Tuxtepec, Oax. México

E-mail: cnolasco@unpa.edu.mx

\section{Luis Felipe Collado}

Institucion: Instituto de Química, Universidad del Papaloapan

Direccíon: Circuito Central 200, Parque Industrial, 68301 San Juan Bautista Tuxtepec, Oax. México

E-mail: jul050794@gmail.com

\section{RESUMEN}

Nuestro planeta se encuentra frente a un fenómeno de contaminación grave que desafortunadamente parece ser ya un proceso integral de nuestra vida cotidiana. El plástico, junto con otros contaminantes sólidos genera toneladas de residuos que ocasionan graves problemas en los ecosistemas acuáticos y terrestres porque no tienen una disposición correcta. Diariamente se escuchan noticias relacionadas con el daño que los plásticos causan a los seres vivos, especialmente a las especies marinas. Uno de los esfuerzos que se realizan para eliminar estos residuos es mediante procesos de reciclado. Dentro del reciclado se destaca la degradación química. El objetivo de este trabajo fue el de aislar e identificar mediante metodologías clásicas y de biología molecular especies microbianas capaces de crecer en una mezcla de compuestos resultantes de una degradación alcalina de plásticos de polietileno (PET). Los resultados muestran que los microorganismos aislados e identificados son en su mayoría hongos y levaduras. Por lo tanto, se iniciaron una serie de estudios tendientes a dilucidar como desarrollan capacidad para metabolizar compuestos resultantes de la degradación alcalina de PET como única fuente de carbono. Se observó que una de las formas de sobrevivir de los hongos y levaduras es mediante la conformación de consorcios microbianos para crecer bajo las condiciones de degradación y de los productos resultantes. En este trabajo se reporta el género y la especie de los hongos aislados. 
Palabras clave: plásticos, hidrolizados de pet, contaminación plástica, consorcios microbianos, reciclado de plásticos, microplásticos.

\begin{abstract}
Our planet is facing a serious pollution phenomenon that unfortunately already seems to be an integral process of our daily life. Plastic, along with other solid pollutants, generates tons of waste that cause serious problems in aquatic and terrestrial ecosystems because they are not properly disposed of. News is heard daily related to the damage that plastics cause to living beings, especially marine species. One of the efforts made to eliminate this waste is through recycling processes. Within recycling, chemical degradation stands out. The objective of this work was to isolate and identify microbial species capable of growing in a mixture of compounds resulting from alkaline degradation of polyethylene plastics (PET) using classical and molecular biology methodologies. The results show that the isolated and identified microorganisms are mostly fungi and yeasts. Therefore, a series of studies were initiated to elucidate how they develop the ability to metabolize compounds resulting from the alkaline degradation of PET as the only carbon source. It was observed that one of the ways of survival of fungi and yeasts is through the formation of microbial consortia to grow under the conditions of degradation and the resulting products. In this work we report the genus and species of the fungi isolated.
\end{abstract}

Keywords: plastics, pet hydrolysates, plastic pollution, microbial consortia, plastic recycling, microplastics.

\title{
1 INTRODUCCIÓN
}

Vivimos rodeados de plástico, un material inventado hace 150 años que supuso una revolución por ser resistente, ligero y económico. Polietileno, poliéster, polipropileno o cloruro de polivinilo están más presentes en nuestro día a día de lo que pensamos: se usan como materiales de construcción, en nuestros vehículos, en el procesado de alimentos y su embalaje, en teléfonos móviles, en la ropa, en la composición de muchos cosméticos e incluso en los utensilios que usamos para comer (Libera, 2019; Rhodes, 2018). Debido a lo anterior, actualmente, en todo el mundo, existe una problemática importante por la contaminación del agua, el aire y el suelo, ocasionada en gran medida, por los grandes volúmenes de residuos que se generan diariamente y que reciben escaso o nulo tratamiento adecuado. Esta situación se agrava porque la basura, que está conformada por residuos de composición muy variada, generalmente se junta y se mezcla durante las labores de recolección lo que dificulta su manejo final. Son numerosos los países que han informado de lo anterior y que se han percatado de los problemas que puede generar este fenómeno como en Perú, país en donde han estudiado la contaminación de las aguas como consecuencia de la contaminación por varios factores, entre ellos los residuos sólidos (Bendezu \& Hernández, 2021).

Recientemente se ha reportado que, a nivel mundial se desperdicia el $75 \%$ de los plásticos que se producen (WWF, 2019). Además, se estima que cada año en promedio 8 millones de toneladas de estos residuos llegan a los diversos mares del mundo trayendo consigo problemas a más de 270 especies marinas, muchas de las cuales terminan enredadas en estos desechos; además de que más de 240 especies 
han ingerido estos plásticos a través de su alimentación y el agua. La industria de los plásticos es un ejemplo del sobreconsumo, debido al uso de múltiples empaques, cada uno de un solo uso en donde los productores enganchan a los consumidores con la idea del reciclaje, pero no indican el procedimiento a seguir o una estrategia de recolección de los envases utilizados. Esto genera que las personas se conviertan en los responsables del destino final de los desechos. Erradicar el patrón cultural de "usar y tirar" es el principal objetivo de la campaña "Océanos Libres de Plásticos" de Greenpeace (Rivera-Garibay et al., 2020).

Otro problema de la contaminación con desechos plásticos vertidos en el mar se relaciona con su fragmentación, para formar microplásticos. Cuando eso sucede, se pueden generar varios fenómenos, el primero es su rompimiento y el segundo su acumulación en el fondo marino. De esta forma podrían ser confundidos por los animales marinos quienes los utilizarán como alimento incluyéndolos, de esta forma, en la cadena alimenticia (Barboza et al., 2018). Se estima que solo en el océano atlántico, en el agua suspendida y en los 200 metros superiores se encuentran entre 11.6 a 21.1 millones de toneladas de microplásticos de polietileno, poliestireno y polipropileno (Pabortsava \& Lampitt, 2020).

El 5 Gyres Institute, órgano consultivo para el Consejo Económico y Social de las Naciones Unidas (ECOSOC por sus siglas en inglés), afirma que existen zonas de acumulación de plástico en cada uno de los cinco giros subtropicales ubicadas en el Pacífico Norte y Sur, el Atlántico Norte y Sur y el Océano Índico. Estas zonas se conocen comúnmente como "islas de plástico”, entre ellas la más grande y conocida es la Gran Mancha de Basura del Pacífico. En 2013 se fundó la organización sin fines de lucro "The Ocean Cleanup" cuyo principal objetivo es limpiar el 50\% de la Gran Mancha de Basura del Pacífico cada 5 años basándose en las corrientes oceánicas. Este sistema ha conseguido capturar 2 toneladas métricas de redes de pesca desechadas, pero no se han obtenido cantidades significantes de otros materiales plásticos ya que el plástico capturado por este sistema solo se retiene pocos días (Libera, 2019). Este es un ejemplo del desarrollo de proyectos a gran escala para tratar de resolver este problema. Sin embargo, se ha reportado que estos proyectos presentan algunos inconvenientes, por ejemplo, este sistema puede capturar algas, medusas y huevos de peces que se encuentran en la superficie del agua pudiendo afectar así a la dieta y poblaciones de ciertas especies.

Adicionalmente, la contaminación de los plásticos en el medio ambiente ha dado lugar a la producción de microplásticos como efecto secundario. Los microplásticos se definen como fragmentos de plástico de menos de $5 \mathrm{~mm}$ (Lim, 2021). Los nano plásticos son incluso más pequeños, con un diámetro de menos de $0.05 \mathrm{~mm}$. La Agencia Europea de Sustancias y Mezclas Químicas (ECHA) plantea, además del tamaño, algunos criterios de identificación de los microplásticos: 1) tipo (materiales poliméricos sintéticos, biopolímeros modificados químicamente), 2) estado (sólido, semisólido) y 3) morfología 
(esferas, fibras, láminas). Las fuentes de microplásticos pueden ser de tipo primario, en donde el microplástico es producido tal cual en la industria cosmética (en la formulación de exfoliantes, pasta de dientes, etc.) o como materia prima para la producción de plásticos (granza) (ECHA, 2019). Las fuentes secundarias incluyen su producción como consecuencia de la degradación física o química de plásticos o fibras de mayor tamaño, que llegan al medio ambiente por una mala gestión de los residuos. Los plásticos, además de las consecuencias sobre el medio ambiente, tienen un efecto directo evidente sobre los seres vivos, ya sea por ingestión, estrangulamiento, atrapamiento o toxicidad (Bollaín-Pastor \& Vicente-Agulló, 2019). Se ha reportado que los microplásticos no están compuestos únicamente de polímeros estructurales (macromoléculas), sino que se pueden considerar como una especie de cocteles complejos de contaminantes, debido a que se ha comprobado que pueden interactuar y sorber fácilmente sustancias toxicas en su superficie, para luego liberarlos. Entre ellas se encuentran aditivos químicos (ftalatos), monómeros residuales y sustancias como retardadores de flama, biocidas e hidrocarburos poliaromáticos (Castañeta et al., 2020; Rojo-Nieto \& Montoto, 2017; WHO, 2019).

Estudios recientes han detectado la presencia de microplásticos en humanos. Lo anterior se basó en un estudio de 47 muestras de tejido humano (corporal, cerebrales, pulmonar, de hígado, de tejido adiposo, de bazo y de riñones) por espectrometría de masas, dando como resultado la presencia de monómeros de diferentes polímeros sintéticos (ACS, 2020; WHO, 2019).

\subsection{PROBLEMA DE LA CONTAMINACIÓN CON POLIETILENTEREFTALATO (PET) EN MÉXICO}

En México el Anuario Estadístico de la Industria Química (ANIQ) reportó la producción, la importación, la exportación y el consumo aparente del polietilentereftalato (PET) entre los años 2010 y 2019. Desde el año 2010, la producción se mantuvo de manera constante en 1000000 de toneladas, mientras que la importación tuvo un aumento de 91000 toneladas en 2019. La exportación aumento 135 000 toneladas y en relación con el consumo aparente hubo una disminución de 112000 toneladas en el año 2019 con respecto al 2010. Estos datos dan fe del papel que ha adquirido este material en nuestras vidas y por ello los problemas ambientales que se han ocasionado a lo largo de su aparición en el mercado mexicano. A nivel mundial México se encuentra entre los países que más desechos de PET producen, principalmente por el al alto consumo de refrescos y agua embotellada. En el año 2017 México se colocó a nivel mundial en la segunda posición en consumo de botellas de PET, en promedio se generan 200 botellas de PET por cada habitante en el país, hoy en día se producen 300 millones de toneladas de plástico de las cuales solo se llega a reciclar el 3\% (Frías et al., 2003; IPN, 2020; Santillán, 2018; Trochillero, 2018). 
Aun siendo México un país en donde se llevan a cabo actividades como acopio y reciclaje de PET, en todo el país solo se recicla el 56\% de las botellas generadas, con excepción de la ciudad de México en donde se recicla el 90\% (López-Casarín, 2019). Ello indica que el resto de PET se encuentra disperso sin tener un procesamiento posconsumo, y que a su vez al llegar a los mares podría generar la formación de microplásticos por fragmentación mecánica en los mares. Como consecuencia de ello se ha reportado su existencia en el estómago de peces en las regiones del Golfo de Baja California Sur, el Golfo de México, Veracruz y en la región del mar Caribe en el Puerto de Morelos (Reyes-Bonilla et al., 2019). Se han reportado de igual forma en Playa Azul, playa Capolita, Zipolite en los estados de Michoacán y Oaxaca (Beltrán et al., 2016; Cruz Salas et al., 2020). De la misma forma científicos del Instituto Politécnico Nacional han realizado estudios de investigación referentes a la contaminación con microplásticos en Huatulco (Oaxaca), la Paz (Baja California), en las playas de Cancún y Tulum y han comenzado estudios en Chetumal (Quintana Roo), Acapulco (Guerrreo) y Tecolutla (Veracruz). Lo anterior se ha realizado para obtener información sobre este problema ambiental y así determinar la afectación a la salud de las personas (IPN, 2020). Lo anterior indica que en México se produce más plástico del necesario y como consecuencia se generan toneladas de basura, que se convierte en un grave problema de contaminación al no poseer nuestro país, un buen sistema de manejo de residuos, tal como lo reportó la Oficina de Información Científica y Tecnológica para el Congreso de la Unión (INCyTU, 2019).

\section{MATERIALES Y MÉTODOS}

\subsection{RECICLAMIENTO Y DEGRADACIÓN DEL PET.}

La degradación resulta un proceso generalmente irreversible que ocasiona un cambio (aunque sea mínimo) en la estructura de un material y que se caracteriza por la pérdida de sus propiedades. Esto puede ocurrir mediante la acción de diversos fenómenos como el calor, la humedad, la radiación solar o de manera enzimática. La degradación de un material sintético como el plástico es un proceso complejo causado por el rompimiento de determinados enlaces químicos originando variaciones tanto físicas como químicas, mismas que se traducen en cambios característicos que se pueden observar a simple vista (Gutiérrez-Pescador, 2013; Madrigal-Cardiel et al., 2022).

En el caso de la degradación de polímeros sintéticos como el PET, esta consiste en romper las cadenas del polímero y formar derivados más cortos que puedan descomponerse o desecharse de manera más fácil. Cuando los fragmentos son degradados y usados como fuente de carbono para la producción de energía por microorganismos, el proceso se denomina biodegradación y el material se considera biodegradable (Yepes-Aguirre, 2014). 
La degradación química es un proceso originado por las reacciones que pueden llevarse a cabo entre algunos reactivos químicos y los polímeros dando como productos diversos compuestos originales o cadenas más cortas del mismo polímero (Posada-Bustamante, 2012). Dentro de estas reacciones se encuentra: a) la hidrólisis, que puede ser ácida, neutra o básica, generando ácido tereftálico y etilenglicol, b) la glicolisis, en donde se generan oligómeros, y c) metanólisis en la que los productos son etilenglicol y dimetil tereftalato. En nuestro caso llevamos a cabo una degradación alcalina generando como producto principal el tereftalato de potasio (Ramírez-Hernández et al., 2010, 2013; Ramírez-Hernández \& NavarroMoreno, 2010). Esa degradación se guardó en un frasco de cristal por varios meses y se observó el crecimiento de algunas formas microbianas. Al llevar a cabo una observación microscópica se pudo comprobar que había estructuras miceliales características de algunos hongos microscópicos. Esta observación fue la que dio vida a presente trabajo de investigación.

\subsection{AISLAMIENTO DE LOS HONGOS CRECIDOS EN UN DEGRADADO QUÍMICO DE PET}

Se tomó una muestra del conglomerado de la solución del degradado de PET (cuyos componentes principales fueron tereftalato de potasio y etilenglicol) realizado en el laboratorio el cual tenía un valor de pH de 12 y se sembró en medio de cultivo Agar Papa Dextrosa (PDA). Se incubo a $37^{\circ} \mathrm{C}$ y se observó su crecimiento cada 24 h registrando sus características morfológicas (tamaño, color y forma de las colonias, tiempo de desarrollo y producción de pigmentos). Una vez que se obtuvieron colonias de hongos separados y limpios se procedió a realizar su identificación.

\subsection{IDENTIFICACIÓN DE LOS HONGOS}

La primera identificación consistió en el análisis de sus características macroscópicas (coloración, morfología, pigmentación, tipo de micelio) y microscópicas (tipo de esporas, hifas y coloración) que presentó cada hongo obtenido. Esta identificación se inició sembrando las muestras aisladas en cajas Petri con medio solido SDA $4 \%$ y dejándolas incubar a $30^{\circ} \mathrm{C}$ por $48 \mathrm{~h}$. Cuando crecieron, se tomaron muestras y se analizaron bajo un microscopio y las observaciones fueron comparadas con la información contenida en la literatura especializada.

La segunda identificación se realizó por la técnica de MALDI-TOF el cual consistió en un análisis proteico generado de los hongos aislados y se realizó la comparación en una base de datos (Cercenado et al., 2003; Clark et al., 2018; Croxatto et al., 2012; Oviaño \& Rodríguez-Sánchez, 2021).

La tercera identificación se realizó por el método de Sanger. Con este método enzimático se determinó la secuencia de DNA. La cuantificación fue llevada a cabo en el equipo NanoDrop (Gauthier, 2008; Sanger et al., 1977; Sikkema-Raddatz et al., 2013). 


\section{RESULTADOS Y DISCUSIÓN}

La Fig. 1 muestra el conglomerado encontrado después de varios meses de crecimiento en el medio alcalino que contenía el degradado de polietilentereftalato, mismo que, posiblemente, fue utilizado como fuente de carbono por este consorcio microbiano. Se puede observar su aspecto algodonoso y de color oscuro en el centro y blanquecino en los bordes. A partir de un examen microscópico se determinó que existían estructuras micelares típicas del crecimiento de los hongos. A partir de una muestra de este material se realizó una siembra en el medio PDA y a partir de esta siembra se aislaron los diferentes microorganismos.

Fig. 1. Conglomerado de microorganismos crecidos en un medio compuesto por un degradado alcalino de polietilentereftalato (Acervo personal de Leticia Navarro, 2019).

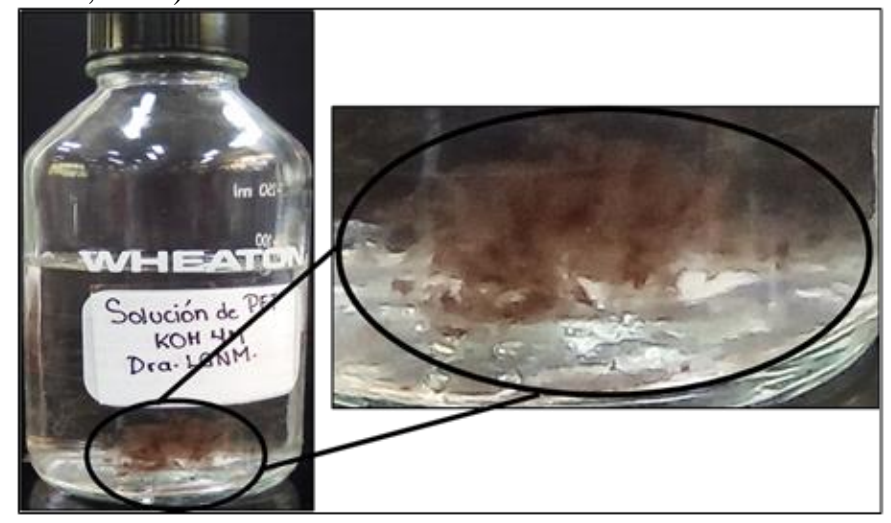

Los diferentes hongos que se lograron aislar a partir del trabajo microbiológico de siembra y resiembra, así como del análisis de sus estructuras microscópicas fueron etiquetados como hongo café, hongo rojo, hongo blanco y hongo negro. Después del análisis microbiológico y empleando las técnicas de biología molecular mostradas en la parte metodológica de este trabajo se pudo identificar el género y la especie de cada uno de ellos. La Tabla 1 muestra el nombre de los microorganismos identificados. Para la identificación morfológica de los hongos se realizó una siembra de los mismos (Hongo café, Hongo rojo, Hongo negro y el Hongo blanco) en medio de cultivo SDA más $4 \%$ de glucosa que constituye un medio más selectivo para el cultivo y aislamiento de levaduras y mohos y por su alta concentración de dextrosa y un bajo $\mathrm{pH}$ que favorece el crecimiento, la formación de esporas y de pigmentos, además de inhibir el crecimiento de las bacterias (Cercenado et al., 2003). Las características macro y microscópicas que presentaron las 4 cepas aisladas se presentan en la Tabla 1 y la Fig. 2. Los hongos se identificaron utilizando las guías reportadas para la identificación de hongos de importancia médica (Larone, 2018) y el "Atlas micológico" del Laboratorio de Microbiología Clínica del Instituto Nacional De Ciencias Médicas y Nutrición Salvador Zubirán. 
Tabla 1. Microorganismos identificados mediante las técnicas microbiológicas y de biología molecular.

\begin{tabular}{ll}
\hline Nomenclatura de los microorganismos & Género y especie identificados \\
\hline Hongo blanco & Acremonium sp \\
Hongo café & Fusarium Proliferatum/Verticillioides \\
Hongo rojo & Talaromyces Verruculosus \\
Hongo negro & Aspergillus Niger \\
\hline
\end{tabular}

\section{Acremonium sp}

Macro morfología: a las 24 h se obtuvieron colonias color blanco tenue con micelio de consistencia algodonosa y al reverso de la caja no presento algún pigmento observable, a las $48 \mathrm{~h}$ solo mostro unos milímetros de crecimiento manteniendo sus características.

Micro morfología: se encontró presencia de esporas en forma de conidios e hifas hialinas no septadas tras ser analizadas en el microscopio a 100x.

\section{Fusarium Proliferatum/Verticillioides}

Macro morfología: a las $24 \mathrm{~h}$ se obtuvo un color blanco tenue en el micelio de consistencia algodonosa y al reverso de la caja no presentó algún pigmento observable. A las 48 h mostró una coloración rosa oscuro con superficie algodonosa de color blanca y rosa, al reverso de la caja presentó una pigmentación rosa a rojizo. Esta característica fue la que se utilizó como información adicional para su identificación.

Micro morfología: se encontró, hifas hialinas macro sifonadas no septadas y abundantes microconidios.

\section{Talaromyces Verruculosus}

Macro morfología: se obtuvieron colonias color crema en la superficie con puntos de color rojo y verde, al reverso presento un tono oscuro con un pigmento rojo.

Micro morfología: se observaron hifas hialinas ramificadas septadas, conidios y esporas en cadena. Por las características macro y microscópicas que el hongo presento se identificó en un principio como Penicillium sp., según lo reportado en la bibliografía usada para la identificación (mencionada anteriormente en la metodología del presente trabajo) y debido a que esta familia es muy parecida al hongo que se deseaba identificar. Sin embargo, tras hacer la identificación por la técnica de secuenciación de Sanger, el organismo se identificó como Talaromyces Verruculosus. 


\section{Aspergillus Niger}

Macro morfología: a las 24 h, presentó un crecimiento de micelio de color amarillo y blanco con el borde negro, a las $48 \mathrm{~h}$ presento crecimiento de esporas negras sobre toda la caja, y al reverso de la caja presentó un color verdoso-amarillento con presencia de anillos.

Micro morfología: se observaron hifas hialinas septadas, conidióforos de cabeza radial extremos ramificados septadas, conidios y esporas en cadena. Luego de utilizar la metodología de MALDI-TOF donde se reconoció como Aspergillus Niger.

Fig. 2. Morfología colonial (izquierda) y microscópica (derecha), observada con un aumento de 40X en un microscopio óptico Olympus CX41. (Fotografías del Acervo personal de Luis Felipe Collado, 2019).

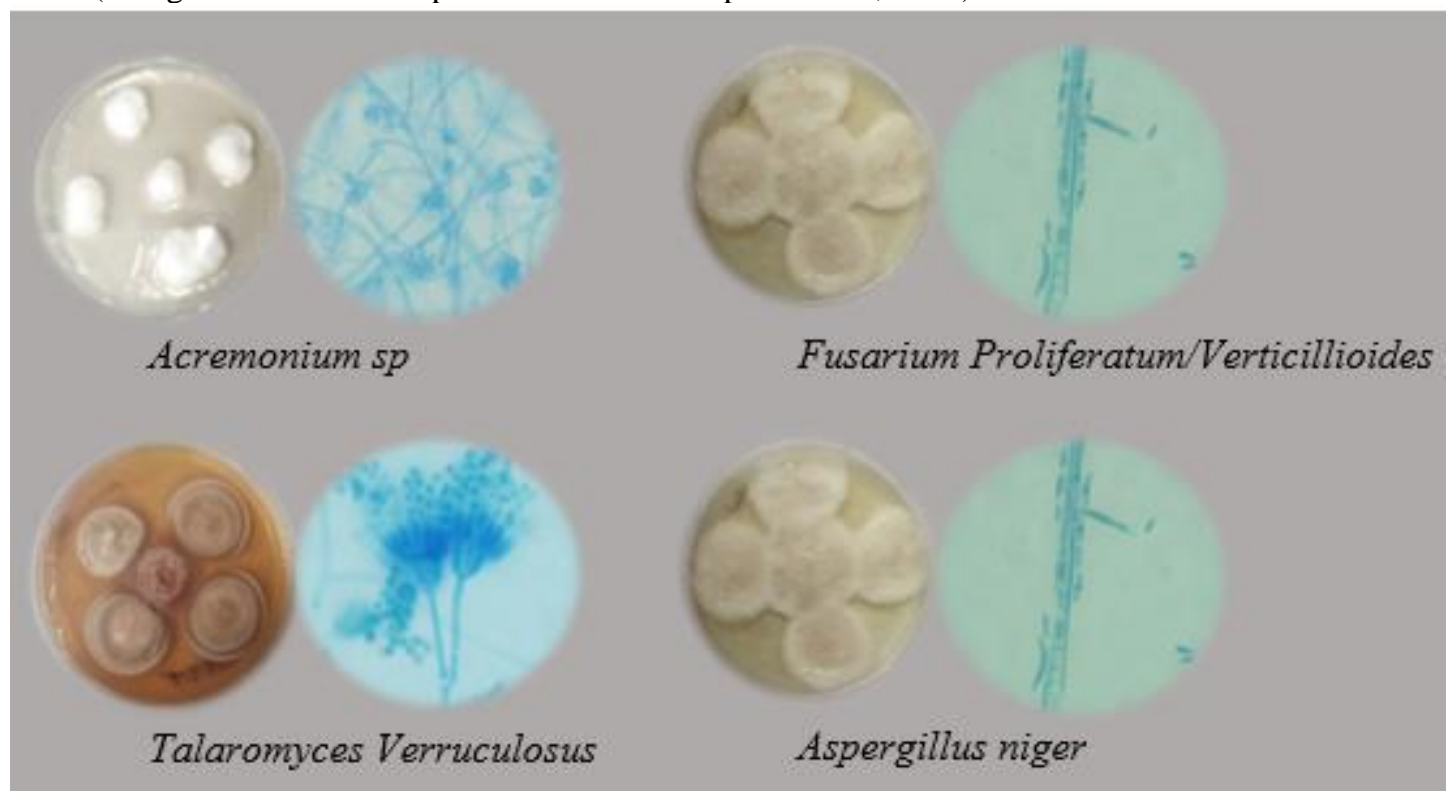

Este escrito muestra una de las partes del trabajo realizado como consecuencia de un trabajo preliminar, en el cual el objetivo fue emplear una técnica de degradación química para tratar de formar un compuesto menos tóxico que el PET (Ramírez-Hernández et al., 2010, 2013; Ramírez-Hernández \& Navarro-Moreno, 2010) sin embargo, al paso del tiempo se observó crecimiento en forma de micelio, el cual llamó la atención debido a las condiciones tanto de los compuestos químicos utilizados en la degradación como del pH al cual esta se llevó a cabo. En la actualidad se sabe que cualquier compuesto puede resultar tóxico y que muchos organismos no pueden vivir a valores de $\mathrm{pH}$ extremos. De la misma manera, se ha reportado la existencia de organismos extremófilos u organismos capaces de adaptarse a condiciones adversas del medio (Ramírez-Duran et al., 2006; Velásquez-Emiliani et al., 2018). En este caso se logró el aislamiento y la caracterización de cuatro hongos. De la misma forma se lograron aislar dos cepas de levaduras. 
En la literatura se han encontrado reportes relacionados con la biodegradación de diferentes compuestos, entre ellos algunos tipos de plásticos y se ha mencionado el nombre de bacterias levaduras y hongos capaces de hacerlo. Un grupo de investigación en México ha reportado la capacidad de los hongos Penicillium pinophilum y Aspergillus niger como posibles microorganismos degradadores de polietileno de baja densidad (Volke-Sepúlveda et al., 2002), así como los microorganismos Aspergillus nomius strain JAPE1 y Streptomyces sp. strain AJ1 reportados previamente (Abraham et al., 2017). Para este tipo de plástico se han establecido diferentes procedimientos de posible biodegradación utilizando condiciones aerobias y anaerobias y recolectando microorganismos de diferentes sitios. Una vez establecidos los protocolos de degradación, este grupo de científicos ha procedido a aislar e identificar varios microorganismos con potencial para la biodegradación (Priyanka \& Archana, 2011). Experimentos similares se han llevado a cabo encontrándose una gran variedad de bacterias que podrían utilizarse como potenciales agentes biodegradables de polietileno (Nowak et al., 2011).

Sin embargo, no existen reportes de microorganismos que degraden, como tal los envases de refresco o de agua, que están formados de polietilentereftalato, debido a la naturaleza química de este polímero o a que las especies microbianas no han desarrollado la maquinaria enzimática para poder romper la matriz polimérica de dichos materiales. En este grupo de investigación se ha observado que, a partir del aislamiento y caracterización de los hongos dos de ellos no pueden crecer en medios de cultivo si no son sembrados junto con los otros microorganismos que forman el consorcio. Esto demuestra la simbiosis que se debió haber establecido como requerimiento para la sobrevivencia de las especies en las condiciones originales de degradación del polímero. Los productos de degradación básica que se obtienen son tereftalato de potasio y el etilenglicol (Ramírez-Hernández et al., 2010) mismos que debieron de servir como fuente de carbono principal para los hongos identificados o, para algunos de ellos, quienes pudieron generar algunos productos secundarios que sirvieran de fuente de carbono a los microorganismos restantes. El estudio de estos hongos podrá servir para establecer relaciones entre microorganismos, procesos de posible biodegradación y fenómenos de resistencia y susceptibilidad de estos frente a diferentes degradados de plásticos. Este punto es importante ya que se relaciona con los fenómenos evolutivos que permiten a los microorganismos adaptarse a ambientes extremos.

\section{CONCLUSIONES}

El estudio de la contaminación ambiental es un tema que abarca un sinfín de subtemas. Entre ellos se encuentran los microorganismos y su capacidad, extraordinaria, de adaptarse a diferentes situaciones extremas como son ambientes con $\mathrm{pH}$ muy alcalino o muy ácido y con sustancias que no se consideran como fuentes de nutrientes. Los microorganismos poseen mecanismos de adaptación especiales que le 
ayudan a desarrollarse y crecer, en este caso, utilizando a un producto de degradación del polietilentereftalato (PET), el tereftalato de potasio o el etilenglicol. Los resultados encontrados en este trabajo indican que los hongos identificados y aislados pueden tener esas propiedades, por lo cual su estudio resulta de gran interés dentro de los campos de la biología, la microbiología, la genética, la ecología, las ciencias ambientales y la biotecnología. Entender los mecanismos que ayudan a los microorganismos a adaptarse podría ser importante para establecer, en un futuro, posibles estrategias de biodegradación de varios compuestos tóxicos que se encuentran contaminando el medio ambiente. 


\section{REFERENCIAS}

Abraham, J., Ghosh, E., Mukherjee, P., \& Gajendiran, A. (2017). Microbial degradation of low density polyethylene. Environmental Progress \& Sustainable Energy, 36(1), 147-154. https://doi.org/10.1002/ep.12467.

ACS. (2020, August 17). Methods for microplastics, nanoplastics and plastic monomer detection and reporting in human tissues. American Chemical Society. News Release. https://www.acs.org/content/acs/en/pressroom/newsreleases/2020/august/micro-and-nanoplasticsdetectable-in-human-tissues.html.

Barboza, L. G. A., Dick Vethaak, A., Lavorante, B. R. B. O., Lundebye, A.-K., \& Guilhermino, L. (2018). Marine microplastic debris: An emerging issue for food security, food safety and human health. Marine Pollution Bulletin, 133, 336-348. https://doi.org/10.1016/j.marpolbul.2018.05.047.

Beltrán, M., Sánchez-García María Elena, Rangel-Alvarez Norma Cristina, Beltrán-Villavicencio Margarita, Vázquez-Morillas Alethia, \& Alvarez-Zeferino Juan Carlos. (2016). Separación y caracterización de micro-plásticos en playas mexicanas. In M. del C. Mañón-Salas (Ed.), Encuentro de Expertos en Residuos Sólidos (pp. 27-33). Sociedad Mexicana de Ciencia y Tecnología Aplicada a Residuos Sólidos, A.C. https://www.researchgate.net/publication/329554229_Separacion_y_caracterizacion_de_microplasticos_en_playas_mexicanas.

Bendezu, M. A. B., \& Hernández, C. V. B. (2021). Efecto de los parámetros fisicoquímicos y biológicos sobre la calidad del agua del río pisco. South Florida Journal of Development, 2(4), 5606-5614. https://doi.org/10.46932/sfjdv2n4-049,

Bollaín-Pastor, C., \& Vicente-Agulló, D. (2019). Presencia de Microplásticos en Aguas y us Potencial Impacto en as Salud Pública. In Rev Esp Salud Pública (Vol. 93). https://scielosp.org/pdf/resp/2019.v93/e201908064/es.

Castañeta, G., Gutiérrez, A. F., Nacaratte, F., \& Manzano, Carlos A. (2020). Microplastics: A Contaminant that Grows in all Environmental Areas, its Characteristics and Possible Risks to Public Health from Exposure. Revista Boliviana de Química, 37(3), 160-175. https://doi.org/10.34098/20783949.37.3.4.

Cercenado, E., Cantón, R., Sánchez, C., Autores, C., Guerrero, C., Carlos, G., \& Carrillo, S. (2003). Procedimientos en Microbiología Clínica Recomendaciones de la Sociedad Española de Enfermedades Infecciosas $\mathrm{y}$ Microbiología Clínica.https://seimc.org/contenidos/documentoscientificos/procedimientosmicrobiologia/seimcprocedimientomicrobiologiala.pdf.

Clark, C. M., Costa, M. S., Sanchez, L. M., \& Murphy, B. T. (2018). Coupling MALDI-TOF mass spectrometry protein and specialized metabolite analyses to rapidly discriminate bacterial function. Proceedings of the National Academy of Sciences, 115(19), 4981-4986. https://doi.org/10.1073/pnas.1801247115.

Croxatto, A., Prod'hom, G., \& Greub, G. (2012). Applications of MALDI-TOF mass spectrometry in clinical diagnostic microbiology. FEMS Microbiology Reviews, 36(2), 380-407. https://doi.org/10.1111/j.1574-6976.2011.00298.x.

Cruz Salas, A., Alvarez Zeferino, J., Martínez-Salvador, C., Enriquez, R., Ojeda-Benitez, S., Vazquez, A., \& Gutiérrez-Ortiz, M. (2020). Cuantificación y caracterización de microplásticos y residuos sólidos 
urbanos en playa Zipolite, Oaxaca. Ciencia y Mar, XXIV(71), 3-21. https://www.researchgate.net/publication/341608868_Cuantificacion_y_caracterizacion_de_microplasti cos_y_residuos_solidos_urbanos_en_playa_Zipolite_Oaxaca.

ECHA, E. C. A. (2019). Annex Xv Restriction Report Proposal for a Restriction. https://echa.europa.eu/documents/10162/05bd96e3-b969-0a7c-c6d0-441182893720.

Frías, A. C., Lema, I. I., \& Gavilán-García Arturo. (2003). La situación de los envases de plástico en México. Gaceta Ecológica, 69, 67-82. https://www.redalyc.org/pdf/539/53906905.pdf.

Gauthier, M. G. (2008). Simulation of polymer translocation through small channels: A molecular dynamics study and a new Monte Carlo approach. http://web5.uottawa.ca/www5/p2uo/website/SlaterTheses/2007-MichelGauthier-PhD.pdf

Gutiérrez-Pescador, J. G. (2013). Degradación de polietileno de baja densidad por consorcios microbianos. Tesis de Grado Facultad de Estudios Superiores Zaragoza. https://www.zaragoza.unam.mx/wpcontent/Portal2015/Licenciaturas/biologia/tesis/tesis_gutierrez_pescador.pdf

INCyTU. (2019, November). Plásticos en los océanos. Información Científica y Tecnológica Para El Congreso de La Unión https://foroconsultivo.org.mx/INCyTU/documentos/Completa/INCYTU_19-034.pdf.

IPN. (2020). Realiza ipn estudios sobre contaminación por microplástico en playas mexicanas. https://www.ipn.mx/assets/files/ccs/docs/comunicados/2020/03/c-041.pdf.

Larone, D. H. (2018). Larone's Medically Important Fungi: A Guide to Identification (T. J. Walsh, R. T. Hayden, \& D. H. Larone, Eds.; 6th Ed.). ASM Press.

Libera. (2019). El impacto del abandono del plástico en la naturaleza. https://proyectolibera.org/wpcontent/uploads/2019/03/Impacto-de-los-plásticos-abandonados_LIBERA-def-1.pdf.

Lim, X. (2021). Microplastics are everywhere — but are they harmful? Nature, 593(7857), 22-25. https://doi.org/10.1038/d41586-021-01143-3.

López-Casarín, J. (2019, October 30). Reciclaje en México. El Economista. https://www.eleconomista.com.mx/opinion/Reciclaje-en-Mexico-20191030-0001.html

Madrigal-Cardiel, M. Á., Velázquez-Roque Silvano, Maldonado-Paleo Jesús, Zurita-Luna, U., \& Hernández-Damián, J. (2022). Diseño y simulación de un molino compacto para polietileno tereftalato. South Florida Journal of Development, 3(1), 232-244. https://doi.org/10.46932/sfjdv3n1-018

Nowak, B., Pająk, J., Drozd-Bratkowicz, M., \& Rymarz, G. (2011). Microorganisms participating in the biodegradation of modified polyethylene films in different soils under laboratory conditions. International Biodeterioration \& Biodegradation, 65(6), 757-767. https://doi.org/10.1016/j.ibiod.2011.04.007.

Oviaño, M., \& Rodríguez-Sánchez, B. (2021). MALDI-TOF mass spectrometry in the 21st century clinical microbiology laboratory. Enfermedades Infecciosas y Microbiologia Clinica (English Ed.), 39(4), 192-200. https://doi.org/10.1016/j.eimce.2020.02.016.

Pabortsava, K., \& Lampitt, R. S. (2020). High concentrations of plastic hidden beneath the surface of the Atlantic Ocean. Nature Communications, 11(1), 4073. https://doi.org/10.1038/s41467-020-17932-9. 
Posada-Bustamante, B. (2012). La degradación de los plásticos. Revista Universidad EAFIT, 67-86. https://publicaciones.eafit.edu.co/index.p.

Priyanka, N., \& Archana, T. (2011). Biodegradability of Polythene and Plastic By The Help of Microorganism: A Way for Brighter Future. Journal of Environmental \& Analytical Toxicology, 01(02). https://doi.org/10.4172/2161-0525.1000111.

Ramírez-Duran, N., Serrano, A. J., \& Sandoval-Trujillo, H. (2006). Revista Mexicana de Ciencias Farmacéuticas. Revista Mexicana de Ciencias Farmacéuticas, 37(3), 56-71. http://www.redalyc.org/articulo.oa?id=57937307.

Ramírez-Hernández, A., Contreras-Crisanto, O., Conde-Acevedo, J., \& Moreno-Navarro, G. L. (2013). Poly( $\varepsilon$-caprolactone) Degradation Under Acidic and Alkaline Conditions. American Journal of Polymer Science, 2013(4), 70-75. https://doi.org/10.5923/j.ajps.20130304.02.

Ramírez-Hernández, A., \& Navarro-Moreno, L. G. (2010). El polietileno, síntesis y degradación. AlephZero-Comprendamos, 57, 1-10. http://www.comprendamos.org/alephzero/57/polietileno.html.

Ramírez-Hernández, A., Navarro-Moreno, L. G., \& Conde-Acevedo, C. (2010). Degradación química del poli(etilen tereftalato). Revista Colombiana de Química, 39(3), 321-331. https://revistas.unal.edu.co/index.php/rcolquim/article/view/20354/21394.

Reyes-Bonilla, H., Alvarez-Filip, L., Rivera Garibay, O., Pérez España, H., Santillo, D., Olivera-Bonilla, A., Pérez-Cervantes, E., \& Rivas-Soto, M. (2019). Estudio sobre el impacto de la contaminación por microplásticos en peces de México. https://www.greenpeace.org/static/planet4-mexicostateless/2019/10/01f918b5-estudio-sobre-el-impacto-de-la-contaminacion-por-microplasticos-en-pecesde-mexico.pdf.

Rhodes, C. J. (2018). Plastic Pollution and Potential Solutions. Science Progress, 101(3), 207-260. https://doi.org/10.3184/003685018X15294876706211.

Rivera-Garibay, O. O., Álvarez-Filip, L., Rivas, M., Garelli-Ríos, O., Pérez-Cervantes, E., \& EstradaSaldívar, N. (2020). Impacto de la contaminación por plástico resumen. https://www.greenpeace.org/static/planet4-mexico-stateless/2020/08/0ead5354-impacto-de-lacontaminacion-por-plastico-resumen.pdf.

Rojo-Nieto, E., \& Montoto, T. (2017). Basuras marinas, plásticos y microplásticos orígenes, impactos y consecuencias de una amenaza global. ISBN:978-84-946151-9-1. Ed. Ecologistas en Acción. https://www.ecologistasenaccion.org/wp-content/uploads/adjuntos-spip/pdf/informe-basurasmarinas.pdf.

Sanger, F., Nicklen, S., \& Coulson, A. R. (1977). DNA sequencing with chain-terminating inhibitors. Proceedings of the National Academy of Sciences, 74(12), 5463-5467. https://doi.org/10.1073/pnas.74.12.5463.

Santillán, M. L. (2018). Una vida de plástico - Ciencia UNAM. Ciencia UNAM. http://ciencia.unam.mx/leer/766/una-vida-de-plastico.

Sikkema-Raddatz, B., Johansson, L. F., de Boer, E. N., Almomani, R., Boven, L. G., van den Berg, M. P., van Spaendonck-Zwarts, K. Y., van Tintelen, J. P., Sijmons, R. H., Jongbloed, J. D. H., \& Sinke, R. J. (2013). Targeted Next-Generation Sequencing can Replace Sanger Sequencing in Clinical Diagnostics. Human Mutation, 34(7), 1035-1042. https://doi.org/10.1002/humu.22332. 
Trochillero. (2018, June 5). El día mundial del medio ambiente, sin contaminación por plástico. El Trochillero.Com. https://eltrochilero.com/plastico-recicla-mexico/.

Velásquez-Emiliani, A. V., Quintero-De La Hoz, M., Jiménez-Vergara, E. Y., Blandón-García, L. M., \& Gómez-León, J. (2018). Microorganismos marinos extremófilos con potencial en bioprospección. Revista de La Facultad de Ciencias, 7(2), 9-43. https://doi.org/10.15446/rev.fac.cienc.v7n2.67360.

Volke-Sepúlveda, T., Saucedo-Castañeda, G., Gutiérrez-Rojas, M., Manzur, A., \& Favela-Torres, E. (2002). Thermally treated low density polyethylene biodegradation by Penicillium pinophilum and Aspergillus niger. Journal of Applied Polymer Science, 83(2), 305-314. https://doi.org/10.1002/app.2245.

WHO. (2019, August 22). WHO calls for more research into microplastics and a crackdown on plastic pollution. WHO News. https://www.who.int/news/item/22-08-2019-who-calls-for-more-research-intomicroplastics-and-a-crackdown-on-plastic-pollution.

WWF. (2019). Solving PlaStic Pollution through accountability. https://www.worldwildlife.org/publications/solving-plastic-pollution-through-accountability.

Yepes-Aguirre, L. M. (2014). Degradación de polietileno de baja densidad utilizando hongos. revisión sistemática de la literatura. Tesis de Licenciatura. Facultad de ciencias. Pontificia Universidad Javeriana. https://repository.javeriana.edu.co/handle/10554/16184/restricted-resource?bitstreamId=455b932f-a3594cd6-8ba5-4625d66a7113. 